UDC 627.152.12

SCOPUS CODE 1900

https://doi.org/10.36073/1512-0996-2019-4-75-81

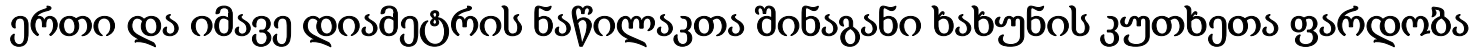

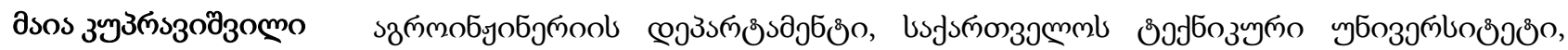

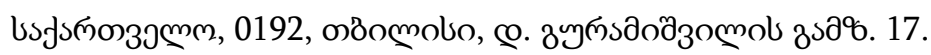

E-mail: m.kupravishvili@gtu.ge

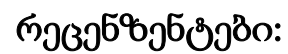

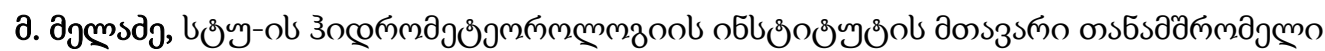

E-mail: m.meladze@gtu.ge

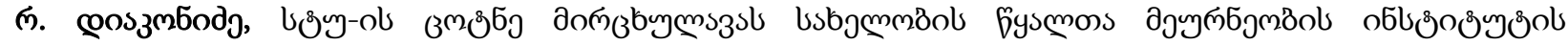

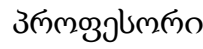

E-mail: robertdia@mail.ru

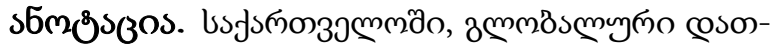

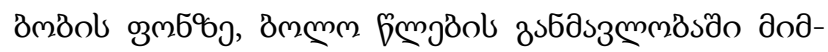

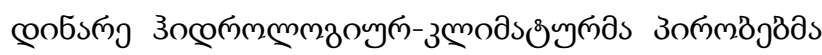

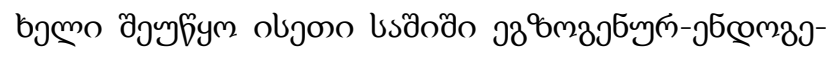

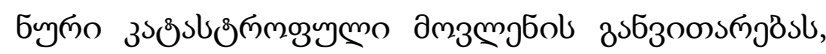

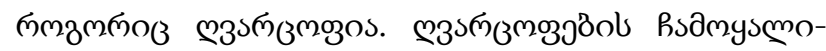

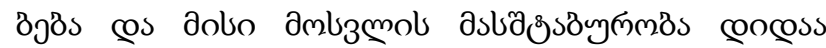

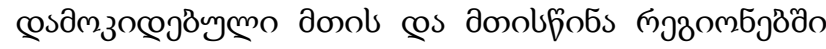

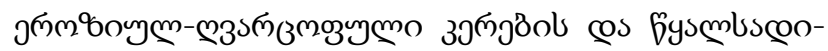

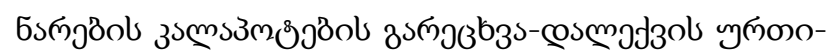

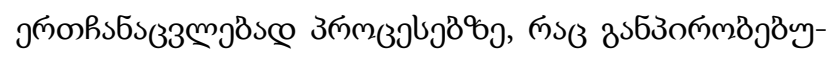

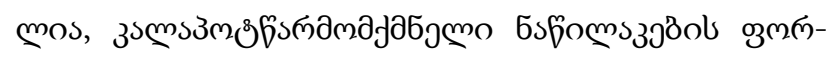

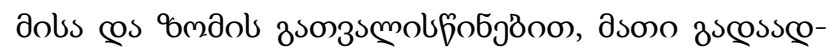

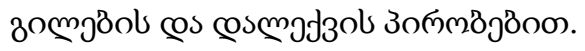

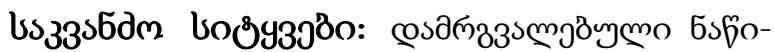

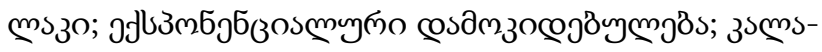

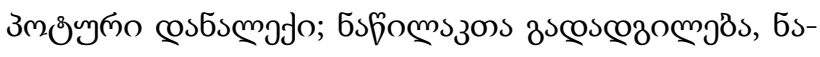

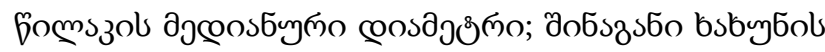

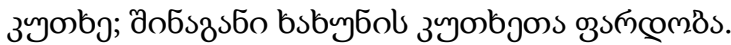

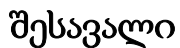

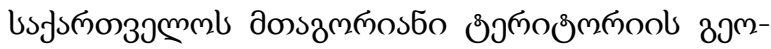

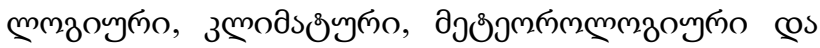

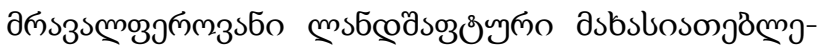

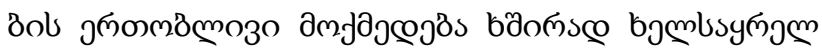

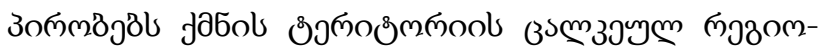

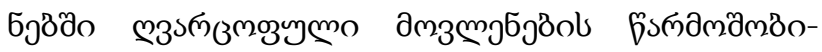

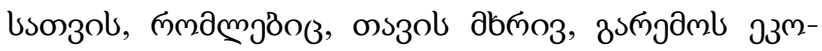




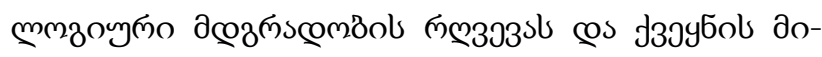

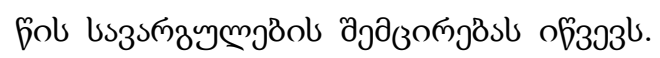

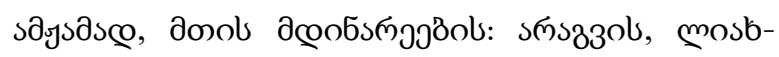

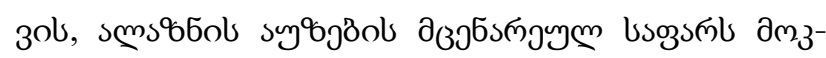

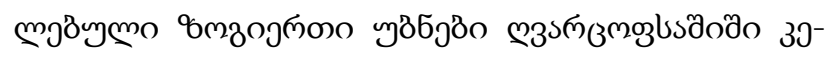

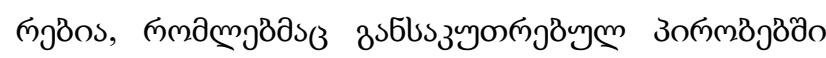

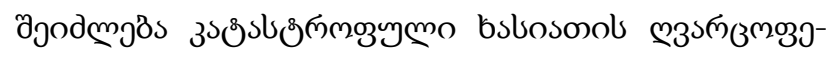

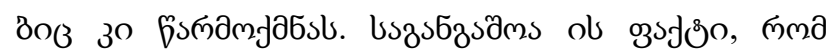

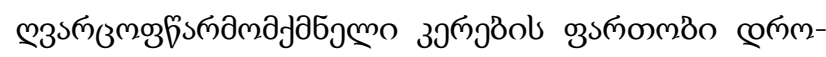

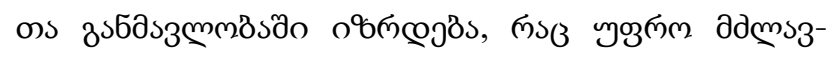

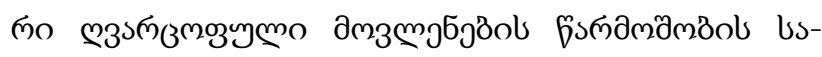

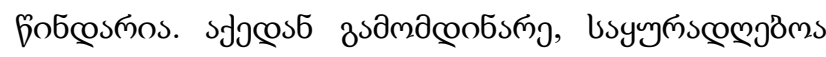

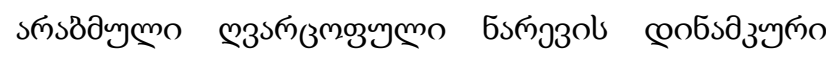

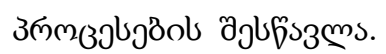

\section{donoossen Esfoemo}

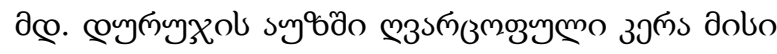

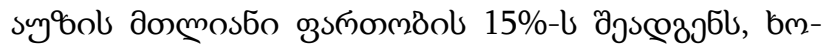

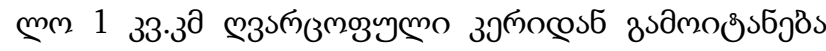

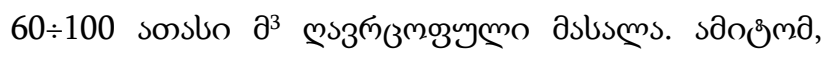

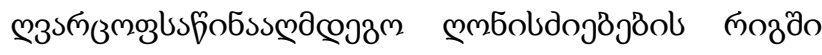

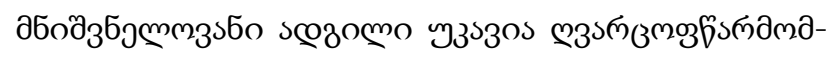

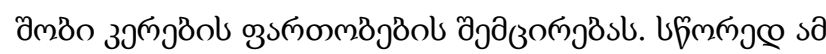

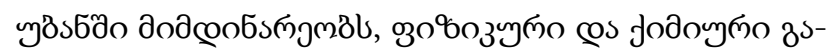

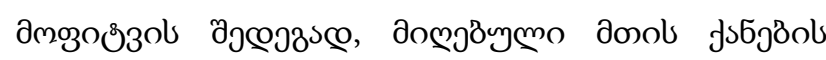

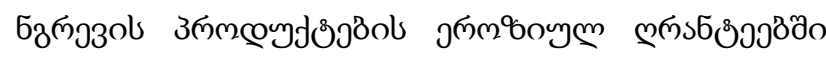

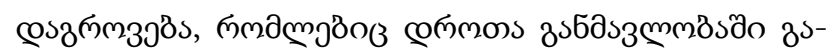

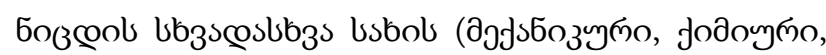

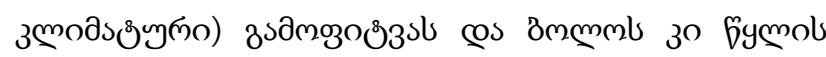

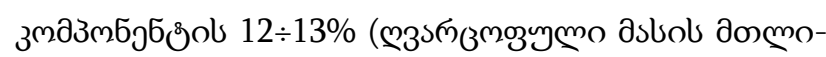

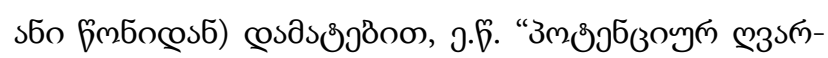

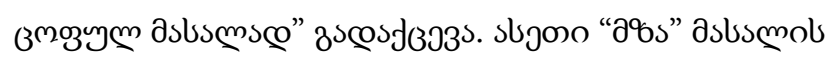

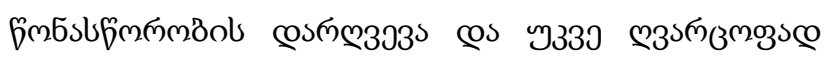

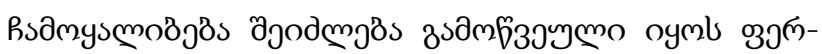

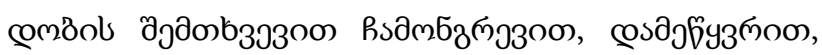

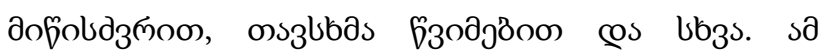

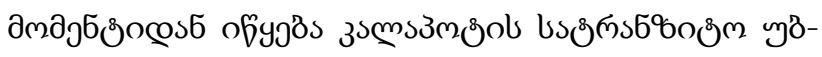

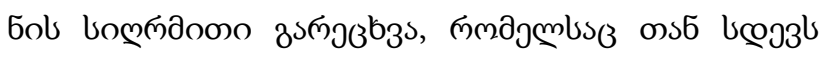

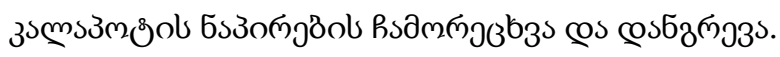

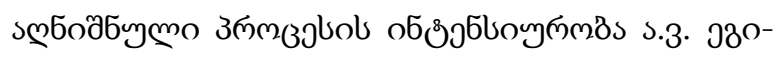

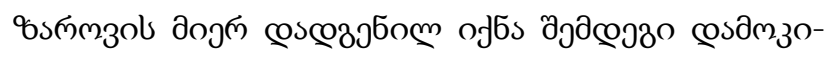

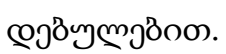

$$
f_{0}=0.06 \cdot \phi \cdot C_{x} \cdot\left(\ell g_{19} \frac{d_{i}}{d_{r}}\right)
$$

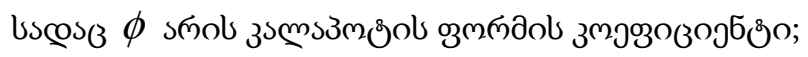

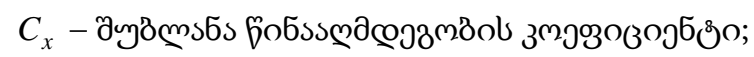

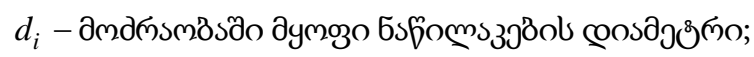

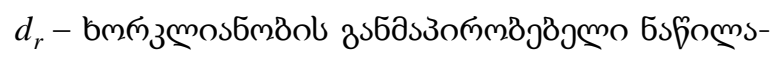

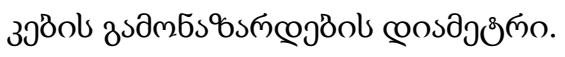

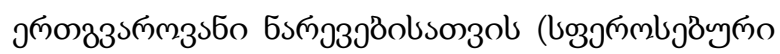

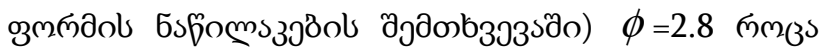

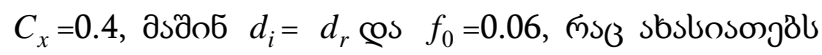

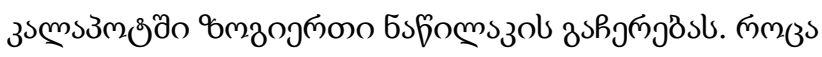

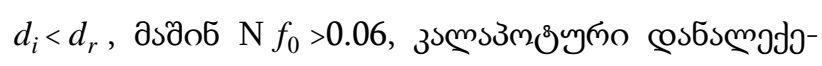

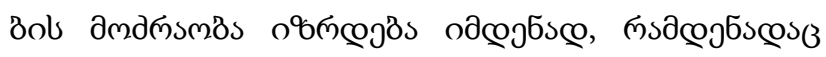

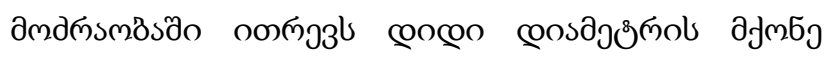

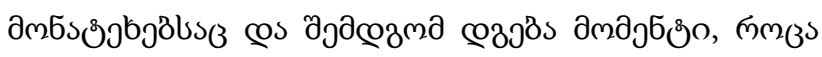

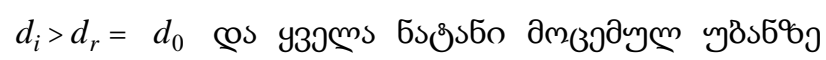

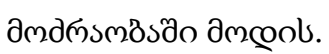

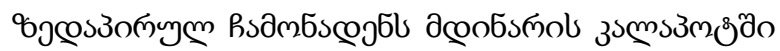

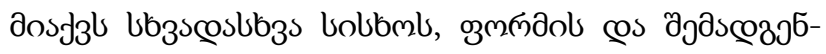

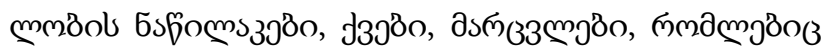

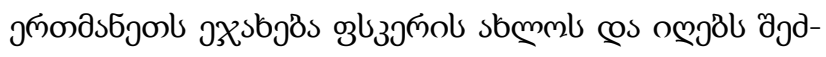




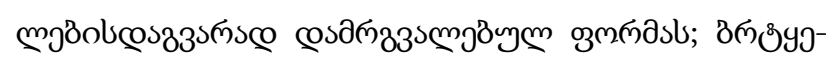

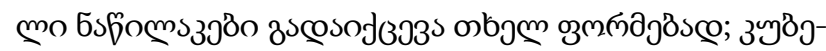

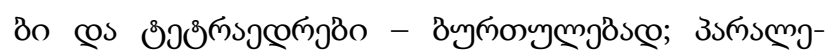

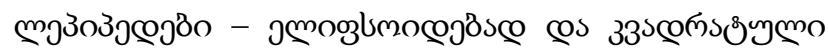

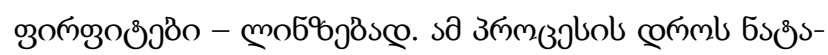

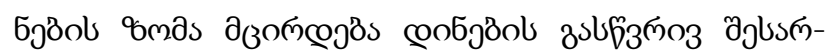

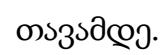

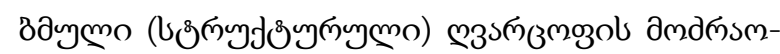

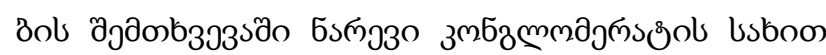

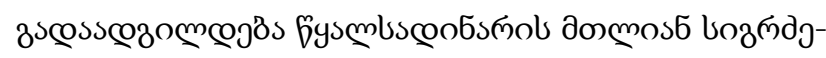

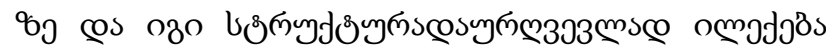

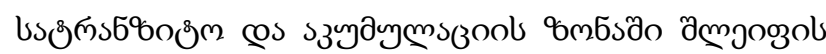

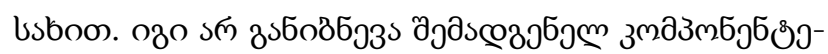

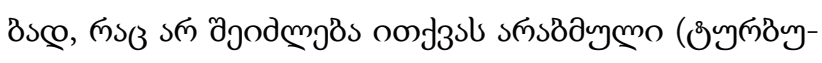

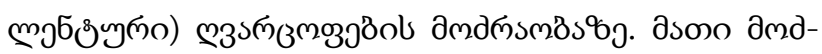

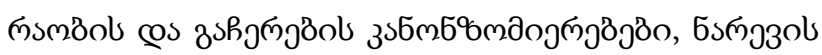

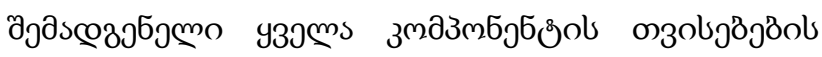

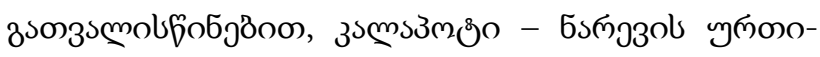

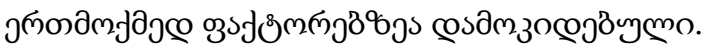

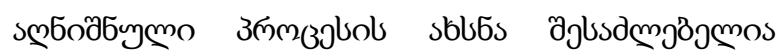

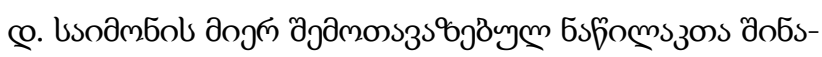

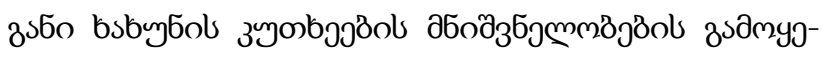
бృ̊̊on.

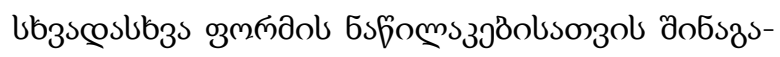

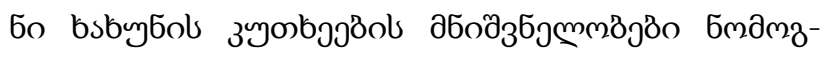

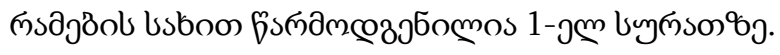

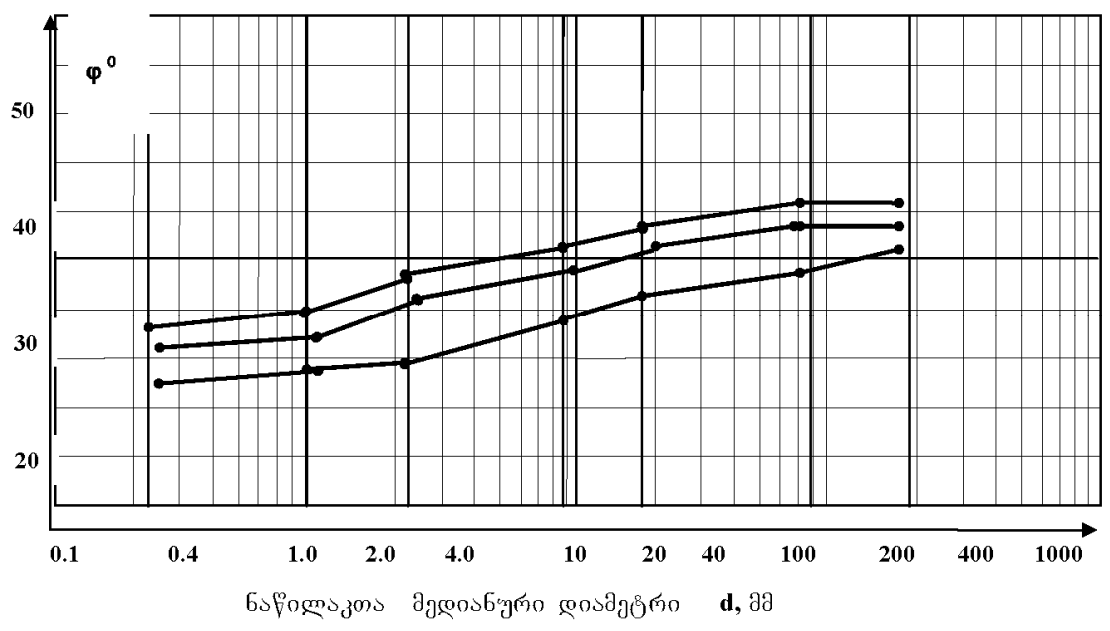

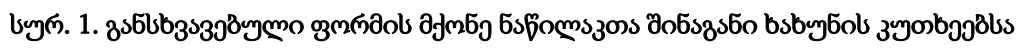

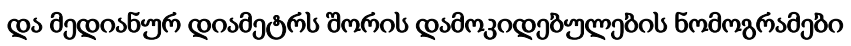

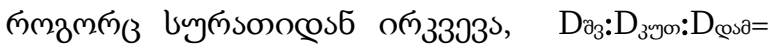

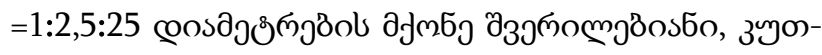

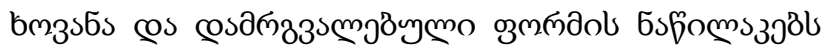

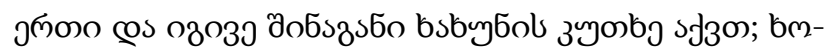

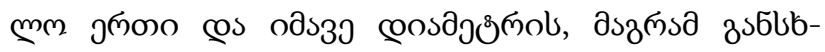

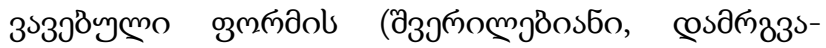

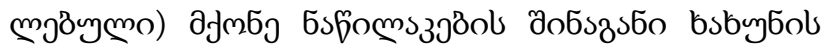

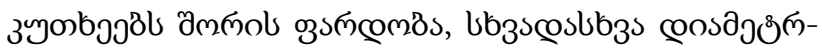

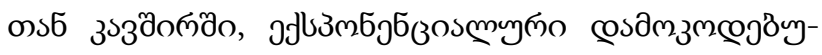

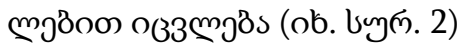




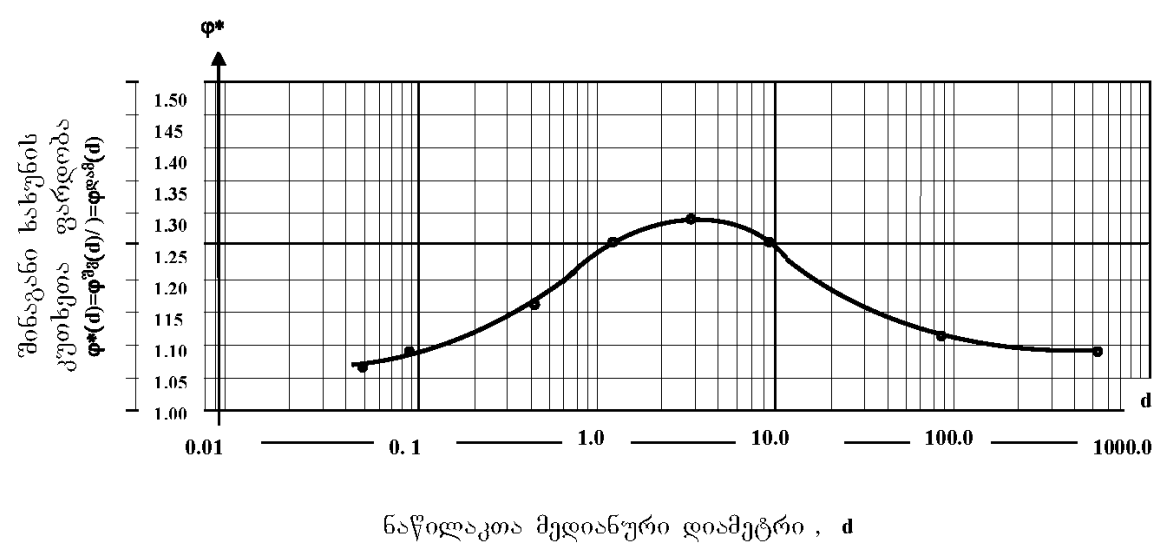

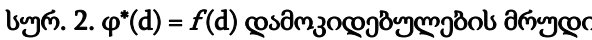

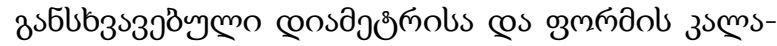

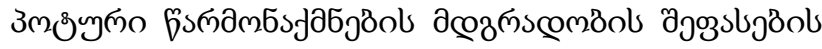

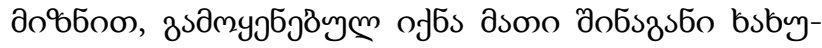

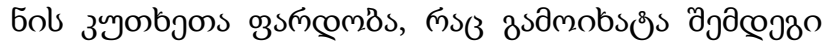
коздмзомублумудоп

$$
\varphi^{*}(d)=1,23 \cdot e^{-(d-6)^{2}}
$$

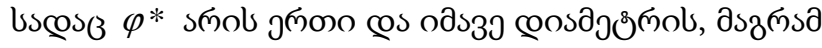

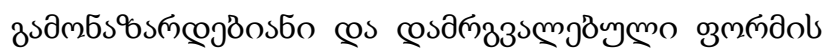

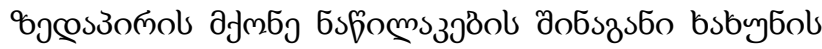

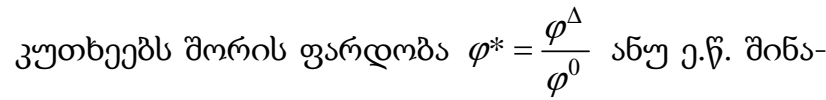

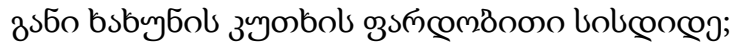

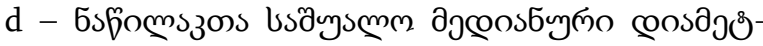
๓о (วд).

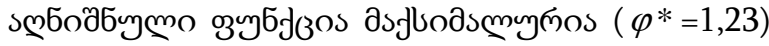

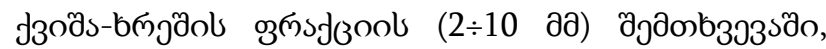

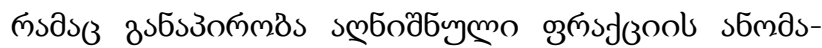

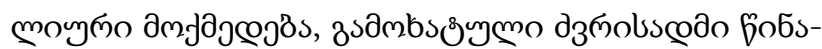

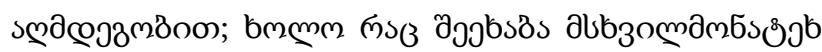

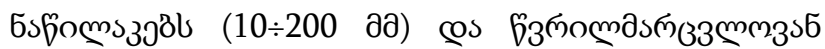

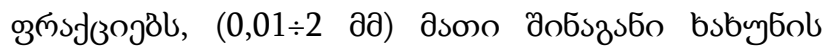

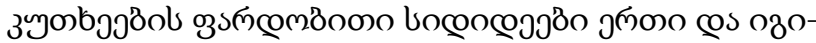

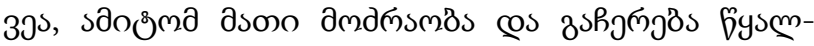

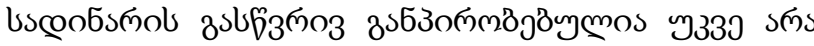

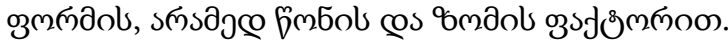

\section{coslos36s}

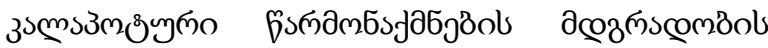

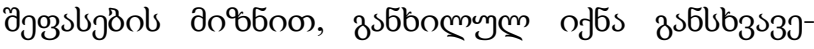

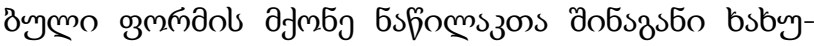

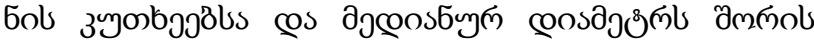

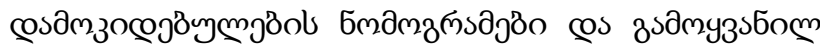

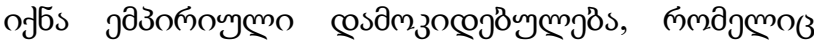

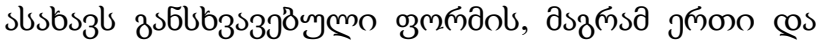

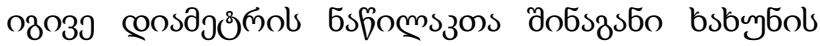

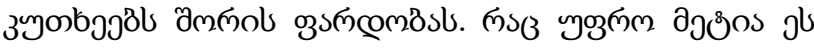

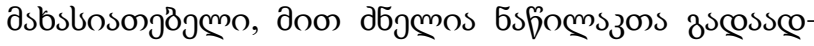

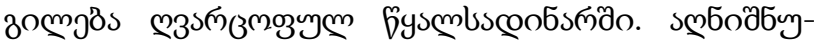

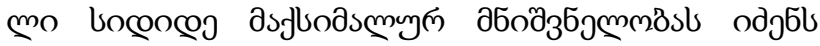

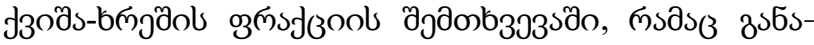

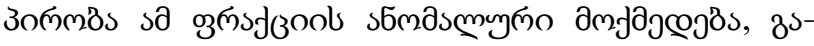

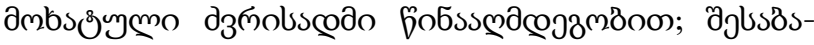




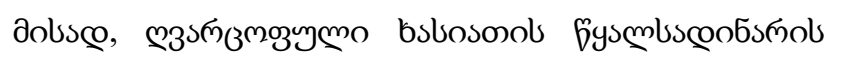

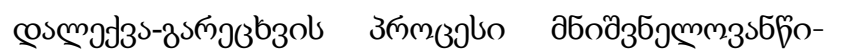

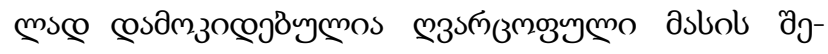

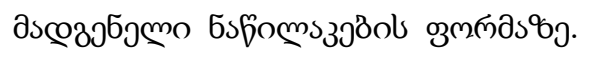

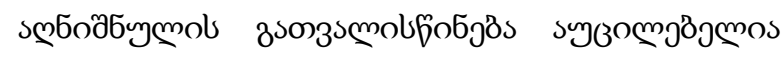

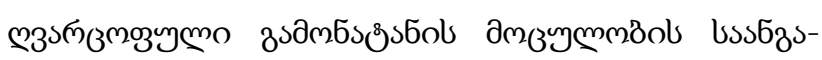

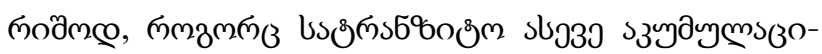

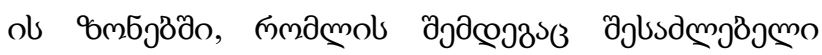

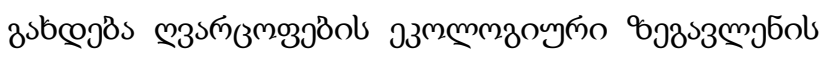

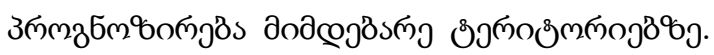

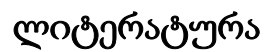

1. Egizarov I.K. To solving the problem of transporting unconnected sediments (any fraction) with influence of their concentration in the layer of the turbidity gradient Iz-vo./ AN. SSSR. 15. 1959. St. 115-126. (in Russian).

2. Egizarov I.K. Traffic heterogeneous sediment. Iz-vo AN. SSSR, seruia. texn. 1963. t-16, 12-3; st.41-50 (in Russian).

3. Kruashvili I.G. Mudflows and methods of struggle with them. monografia, Tbilisi, 2014, 337 gv. (in Georegain).

4. Kruashvili I.G., Kukhalashvili E.G., Inashvili I.D., Bziava K.G. Debris flow phenomena. Risk, Prediction, Protection. monografia. saqartvelos teqnikuri universiteti, tbilis, 2017, 249 gv.(in Georgian).

5. Natishvili O.G., Kruashvili I.G. Protection of objects from the harmful effects of mudflows. J. Ekologicheskie sistemi I pribori, \#7, 2015, st.20-30. (in Russian).

6. Siamashvili A.R. Impact accounting forms of cross section of the channels in establishing permissible (nonwashable) speeds of flows. Soob. ANSSSR. 1986. 1123 (2). st. 353-356. (in Russian).

7. Simons D.B. Sediment transport technology. Water Resorces Public. Fort. Collins. 1977. (in English). 
UDC 627.152.12

SCOPUS CODE 1900

\title{
Correlation of internal friction angles of the particles with the same diameters
}

\author{
Maia Kupravishvili Department of Agro-Engineering, Georgian Technical University, 17 \\ D. Guramishvili Str, 0192, Tbilisi, Georgia \\ E-mail: m.kupravishvili@gtu.ge
}

\section{Reviewers:}

M. Meladze, Chief Specialist, Georgian Technical University Institute of Hydrometeorology

E-mail: m.meladze@gtu.ge

R. Diakonidze, Professor, Tsotne Mirtskhulava Water Management Institute of the Georgian Technical University

E-mail: robertdia@mail.ru

Abstract. Nomograms of the relationship between the internal friction angles and the median diameters of the particles of different shapes have been considered in order to evaluate the stability of the channel forming particles.

Based on experimental and theoretical studies, we have obtained correlations between internal friction angles of the particles of different particle shapes with the same diameters - the larger this indicator, the less probability the bottom sediments are to move and vice versa. This correlation acquires the maximum value in the case of sandgravel fraction, which led to its anomalous action. It has been ascertained, that the washing and sedimentation process of the water channel of the debris flow largely depends on the shape of its constituting particles.

It is desirable to take the above mentioned into account when calculating the volumes of mudflow in the transit and accumulation zones, after which it will be possible to predict the ecological impact of the mudflows on the adjacent area.

Key words: Channel-forming particles; correlation of internal friction angles of the particles; exponential dependence; internal friction angle; particle median diameters; particle motion; rounded particle. 
UDC 627.152.12

SCOPUS CODE 1900

\section{Соотношение углов внутреннего трения частиц с одинаковыми диаметрами}

Мая Куправишвили Департамент Агроинженерии, Грузинский Технический Университет, Грузия, 0192, Тбилиси. пр. Д. Гурамишвили 17

E-mail: m.kupravishvili@gtu.ge

\section{Рецензенты:}

М. Меладзе, главный сотрудник Института гидрометеорологии ГТУ

E-mail: m.meladze@gtu.ge

Р. Диаконидзе, профессор водного хозяйства Института Цотне Мирцхулава ГТУ

E-mail: robertaia@mail.ru

Аннотация. Чтобы оценить устойчивость руслообразующих частиц были рассмотрены номограммы зависимости между углами внутреннего трения и срединными диаметрами разных форм частиц. При экспериментальных и теоретических исследованиях были получены соотношения между углами внутреннего трения различных форм частиц, при одинаковых диаметрах - чем больше этот показатель, тем меньше вероятность передвижения донных наносов, и наоборот. Это соотношение приобретает максимальное значение в случае песчано-гравийной фракции, что привело его к аномальному действию, выраженному сопротивлением устойчивости этих частиц. Следовательно, процесс динамического заиления-размыва русла преимущественно зависит от показателей формы составляющих его частиц.

Желательно вышеуказанное учитывать при расчёте объемов селевого потока как в транзитной, так и в аккумуляционной зонах, после чего можно будет прогнозировать экологическое влияние селевых потоков на прилегающую территорию.

Ключевые слова: движение частиц; округленная частица; руслообразующие частицы; соотношение углов внутреннего трения частиц; средний диаметр; угол внутреннего трения; экспоненциальное отношение.

\footnotetext{
8s6bocrzol onsณомо 28.06.2019

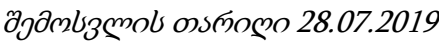

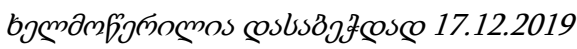

